\title{
BMJ Open Instruments for assessing health professionals' skills in breaking bad news: protocol for a systematic review of measurement properties
}

\author{
Daniel Gutierrez-Sanchez, ${ }^{1}$ Marina García-Gámez (D) , ${ }^{2}$ Juan Pablo Leiva-Santos, ${ }^{3}$ \\ Inmaculada Lopez-Leiva ${ }^{1}$
}

To cite: Gutierrez-Sanchez D, García-Gámez M, LeivaSantos JP, et al. Instruments for assessing health professionals' skills in breaking bad news: protocol for a systematic review of measurement properties. BMJ Open 2021;11:e048019. doi:10.1136/ bmjopen-2020-048019

- Prepublication history for this paper is available online. To view these files, please visit the journal online (http://dx.doi. org/10.1136/bmjopen-2020048019).

Received 15 December 2020 Accepted 15 July 2021

\section{Check for updates}

\section{Author(s) (or their} employer(s)) 2021. Re-use permitted under CC BY-NC. No commercial re-use. See rights and permissions. Published by BMJ.

${ }^{1}$ Faculty of Health Sciences, Department of Nursing, University of Málaga, Málaga, Spain, Biomedical Research Institute of Málaga (IBIMA)

Málaga, Andalucía, Spain

${ }^{2}$ Faculty of Health Sciences, Department of Nursing, University of Málaga, Malaga, Andalucía, Spain

${ }^{3}$ Hospital de Manacor, Carretera de Manacor-Alcudia, Mallorca, Islas Baleares, Spain

Correspondence to Dr Marina García-Gámez; magagadue@uma.es

\section{ABSTRACT}

Introduction Health professionals are often involved in the process of breaking bad news (BBN), which remains a difficult challenge, as it requires not only theoretical knowledge, but also the development of humanistic, emotional and communication skills. Therefore, optimal BBN assessment is essential. In this regard, sound measurement instruments are needed to evaluate BBN properly in research, teaching and clinical settings. Several instruments have been designed and validated to assess BBN. In this context, choosing the most appropriate instrument for assessing health professionals' skills in BBN is essential. The aims of this systematic review are to: (1) identify all the instruments used for assessing health professionals' skills in BBN; and (2) critically appraise their measurement properties.

Methods A systematic review will be undertaken according to the most up-to-date COnsensus-based Standards for the selection of health status Measurement INstruments' (COSMIN) methodology. The protocol of this systematic review was developed using the Preferred Reporting Items for Systematic Reviews and Meta-Analyses guidelines. The search strategy will be performed following the Peer Review of Electronic Search Strategies. The search strategy will be conducted in CINAHL, MEDLINE, Embase, PsycINFO, SciEL0 and Open Grey. Two review authors will independently appraise the full-text articles according to the COSMIN Risk of Bias checklist. Quality ratings and evidence synthesis will be performed using a modified Grading of Recommendations Assessment, Development and Evaluation approach. Ethics and dissemination Ethical approval is not necessary for systematic review protocols. The results will be disseminated by publication in a peer-reviewed journal and presented at a relevant conference.

PROSPERO registration number CRD42020207586.

\section{INTRODUCTION}

Bad news is any information that changes a person's view of the future in a negative way. ${ }^{12}$ Although breaking bad news (BBN) is frequently viewed as a process that is important for physicians working in endof-life care, most health professionals break bad news on different levels with different
Strengths and limitations of this study

- This will be the most up-to-date systematic review identifying and critically appraising the measurement properties of instruments assessing health professionals' skills in breaking bad news (BBN) according to the Consensus-based Standards for the selection of health status Measurement INstruments' methodology.

- This systematic review protocol was developed according to the Preferred Reporting Items for Systematic Reviews and Meta-Analyses guidelines.

- A limitation of this systematic review is the inclusion of articles published only in English and Spanish, which may lead to language bias. To reduce potential language bias, studies using translated versions of instruments assessing BBN will be included.

types of patient populations. ${ }^{34}$ In addition, health professionals are often involved in the process of BBN, but it remains a difficult challenge for them. ${ }^{56}$ In this context, different elements from health professions such as a lack of ability in dealing with own feelings as well as the listener's reaction, or a lack of communication skills make the BBN process difficult. ${ }^{78}$ Accordingly, the complexity of communicating bad news lies in numerous factors, such as the health professional's response to the emotional reactions of patients and their caregivers, who may feel fearful or unable to cope with the situation, which can result in emotional reactions that include shock, anger, sadness, denial, relief and acceptance; as well as managing ones' own emotional reactions to bad news. ${ }^{9}$ Therefore, delivering BBN requires not only theoretical knowledge, but also the development of a series of humanistic, emotional and communication skills. ${ }^{379}$ In this regard, focused training in communication skills and techniques to facilitate $\mathrm{BBN}$ has been 
demonstrated to improve patient satisfaction and health professionals comfort. ${ }^{1011}$

The assessment of the BBN process is influenced by psychological and emotional factors from both sides of the communication: the patient and family, and the healthcare professional. ${ }^{2312}$ In this regard, BBN is difficult to evaluate and one of the most feared processes for professionals and patients. Although different measures have been developed for evaluating this construct, there is little evidence of assessment of the BBN process, and therefore instruments to measure $\mathrm{BBN}$ are needed, as assessment of the $\mathrm{BBN}$ process is increasingly used in research, teaching and clinical settings. ${ }^{72-16}$ In this context, choosing the most appropriate instrument for assessing BBN is challenging, and the choice should be dependent on the measurement properties and feasibility of the instruments. ${ }^{17}$ In this regard, previous systematic reviews on BBN assessment have been performed. ${ }^{16}{ }^{18}$ In this context, Singh and Agarwal carried out a systematic review which identified and described different protocols for BBN and Johnson and Panagioti conducted a systematic review and meta-analysis on interventions to improve BBN by physicians and medical students. However, although previous systematic reviews on BBN have been performed, no methodologically sound review has been published that contrasts the measurement properties of all the instruments assessing the BBN process according to the most up-to-date COnsensus-based Standards for the selection of health status Measurement INstruments' (COSMIN) methodology. ${ }^{19}$

Therefore, the aim of this systematic review is twofold: (1) to identify all the instruments that have been used to evaluate the BBN process; and (2) to critically appraise the measurement properties of all these instruments.

\section{METHODS}

\section{Objectives}

This systematic review aims to: (1) identify the measures assessing BBN in the healthcare setting; and (2) critically appraise the methodological quality of studies on measurement properties.

\section{Design}

The protocol of this study was developed according to the Preferred Reporting Items for Systematic Reviews and Meta-Analyses Protocols (PRISMA-P) and was registered in the International Prospective Register of Systematic Reviews (PROSPERO) ${ }^{20}$

The systematic review will be carried out according to the most up-to-date COSMIN methodology. ${ }^{19}$ In this context, the COSMIN initiative aims to improve the selection of health measurement instruments in research and clinical practice by developing tools and guidelines to select the most appropriate instrument for a given purpose.

\section{Search strategy}

Searches will be conducted in MEDLINE, PsycINFO, CINAHL, Cochrane Library, Web of Science, Scopus,
COSMIN database of systematic reviews and Open Grey following the Peer Review of Electronic Search Strategies. ${ }^{21}$

The search strategy will include a combination of the following aspects:

- Construct search.

- Population search.

- Instruments search.

- Filter for psychometric properties.

- Exclusion filter.

The exclusion filter will be mainly limited to publication types and subject groups. A search strategy has been proposed (table 1). Moreover, other relevant papers may be identified by checking the reference lists of included articles.

The search strategy will be limited to articles published in English and Spanish.

\section{Inclusion and exclusion criteria}

Eligible studies will be those that satisfy the following inclusion criteria: (a) all study designs which used instruments assessing BBN in a healthcare setting; (b) assessing at least one measurement property of an instrument for measuring BBN; (c) published in English or Spanish; (d) all publication years.

Biographies, addresses, editorials, case reports, comments and congresses, conferences, practice guidelines and articles not published in the English or Spanish languages will be excluded.

\section{Outcomes}

All measurement properties of the instruments assessing BBN will be considered as outcomes. The COSMIN taxonomy will be adopted for the measurement properties of the instruments.

\section{Study screening and selection}

Titles and abstracts will be screened independently by two review authors. All identified references will be entered into Mendeley bibliographic software and recorded duplicates removed. The full article will be retrieved when decisions cannot be made based on the abstract and title alone. Any disagreements between the two reviewers will be resolved by a third reviewer, as necessary.

A PRISMA flow diagram for this systematic review will be provided.

\section{Quality appraisal}

To evaluate the quality of each study as a whole, a specific reporting guideline will be used, depending on the study design. The methodological quality of each study for each measurement property will be assessed according to the COSMIN Risk of Bias checklist.

Two review authors will independently appraise the full-text articles (DGS and JPLS) according to the most up-to-date COSMIN Risk of Bias checklist with a fourpoint scale, and disagreements will be resolved by a third reviewer. ${ }^{22}$ This tool considers 10 measurement properties. A methodological quality score is provided for the 
Table 1 Search strategy

1 "Communicating bad news" [tiab] OR "Breaking bad news" [tiab] OR "Delivering bad news" [tiab] OR "Difficult news" [tiab] OR "Truth disclosure" [MeSH] AND "Communication skills" [tiab] OR "Communication abilities" [tiab]

2 "Health professional" [tiab] OR "Healthcare professional" [tiab] OR "Health workers" [tiab] "Healthcare workers" [MeSH] OR "Health staff" [tiab] OR "Healthcare staff" [tiab] OR "Health personnel" [MeSH] "Healthcare providers" [MeSH]

3 (Instrument[tiab] OR instruments[tiab] OR measure [tiab] OR measures [tiab] OR questionnaire[tiab] OR questionnaires[tiab] OR scale[tiab] OR scales[tiab] OR tool[tiab] OR tools[tiab] OR survey [tiab] OR test [tiab]

4 Instrumentation[sh] OR methods[sh] OR "Validation Studies"[pt] OR "Comparative Study"[pt] OR "psychometrics"[MeSH] OR psychometr*[tiab] OR clinimetr*[tw] OR clinometr*[tw] OR "outcome assessment (health care)"[MeSH] OR "outcome assessment"[tiab] OR "outcome measure *"[tw] OR "observer variation"[MeSH] OR "observer variation"[tiab] OR "Health Status Indicators"[Mesh] OR "reproducibility of results"[MeSH] OR reproducib*[tiab] OR "discriminant analysis"[MeSH] OR reliab*[tiab] OR unreliab*[tiab] OR valid*[tiab] OR coefficient[tiab] OR homogeneity[tiab] OR homogeneous[tiab] OR "internal consistency"[tiab] OR (cronbach*[tiab] AND (alpha[tiab] OR alphas[tiab])) OR (item[tiab] AND (correlation*[tiab] OR selection*[tiab] OR reduction*[tiab])) OR agreement[tiab] OR precision[tiab] OR imprecision[tiab] OR "precise values"[tiab] OR test-retest[tiab] OR (test[tiab] AND retest[tiab]) OR (reliab* [tiab] AND (test[tiab] OR retest[tiab])) OR stability[tiab] OR interrater[tiab] OR inter-rater[tiab] OR intrarater[tiab] OR intra-rater[tiab] OR intertester[tiab] OR inter-tester[tiab] OR intratester[tiab] OR intratester[tiab] OR interobserver[tiab] OR inter-observer[tiab] OR intraobserver[tiab] OR intraobserver[tiab] OR intertechnician[tiab] OR inter-technician[tiab] OR intratechnician[tiab] OR intra-technician[tiab] OR interexaminer[tiab] OR inter-examiner[tiab] OR intraexaminer[tiab] OR intra-examiner[tiab] OR interassay[tiab] OR inter-assay[tiab] OR intraassay[tiab] OR intra-assay[tiab] OR interindividual[tiab] OR inter-individual[tiab] OR intraindividual[tiab] OR intra-individual[tiab] OR interparticipant[tiab] OR inter-participant[tiab] OR intraparticipant[tiab] OR intra-participant[tiab] OR kappa[tiab] OR kappa's[tiab] OR kappas[tiab] OR repeatab*[tiab] OR ((replicab*[tiab] OR repeated[tiab]) AND (measure[tiab] OR measures[tiab] OR findings[tiab] OR result[tiab] OR results[tiab] OR test[tiab] OR tests[tiab])) OR generaliza*[tiab] OR generalisa*[tiab] OR concordance[tiab] OR (intraclass[tiab] AND correlation*[tiab]) OR discriminative[tiab] OR "known group"[tiab] OR "factor analysis"[tiab] OR "factor analyses"[tiab] OR dimension*[tiab] OR subscale*[tiab] OR (multitrait[tiab] AND scaling[tiab] AND (analysis[tiab] OR analyses[tiab])) OR "item discriminant"[tiab] OR "interscale correlation*"[tiab] OR error[tiab] OR errors[tiab] OR "individual variability"[tiab] OR (variability[tiab] AND (analysis[tiab] OR values[tiab])) OR (uncertainty[tiab] AND (measurement[tiab] OR measuring[tiab])) OR "standard error of measurement" [tiab] OR sensitiv*[tiab] OR responsive*[tiab] OR ((minimal[tiab] OR minimally[tiab] OR clinical[tiab] OR clinically[tiab]) AND (important[tiab] OR significant[tiab] OR detectable[tiab]) AND (change[tiab] OR difference[tiab])) OR (small*[tiab] AND (real[tiab] OR detectable[tiab]) AND (change[tiab] OR difference[tiab])) OR "meaningful change" [tiab] OR "ceiling effect"[tiab] OR "floor effect"[tiab] OR "Item response model"[tiab] OR IRT[tiab] OR Rasch[tiab] OR "Differential item functioning"[tiab] OR DIF[tiab] OR "computer adaptive testing"[tiab] OR "item bank"[tiab] OR "cross-cultural equivalence"[tiab]

$5 \quad$ \#1 AND \#2 AND \#3 AND \#4

6 "Protocol" [Publication Type] OR "addresses"[Publication Type] OR "biography"[Publication Type] OR "case reports"[Publication Type] OR "comment"[Publication Type] OR "editorial"[Publication Type] OR "congresses" [Publication Type] OR "consensus development conference"[Publication Type] OR "consensus development conference"[Publication Type] OR "practice guideline"[Publication Type]) OR "suffering from"[tiab] OR "animals"[MeSH]

$7 \quad$ \#5 NOT \#6

8 FILTER: Language (English and Spanish)

following measurement properties: instrument development; content validity; structural validity; internal consistency; cross-cultural validity/measurement invariance; reliability; measurement error; criterion validity; hypotheses testing for construct validity; and responsiveness. Each measurement property contains 3-35 items and each item has four response options: 'very good'; 'adequate'; 'doubtful'; or 'inadequate'. The overall methodological quality score of each measurement property is obtained based on the worst score counts' method. ${ }^{22}$

\section{Data extraction}

Two researchers will independently participate in the data extraction process, and any disagreements will be resolved by a third reviewer. The following information will be extracted: study author and date of publication; country and language; study population; sample size; setting; instrument description; measurement properties; COSMIN score; and measurement values.

\section{Data synthesis}

Data synthesis will comprise three steps. First, the methodological quality of each study that meets the inclusion criteria will be rated using the COSMIN Risk of Bias checklist with quality ratings as either very 'good', 'adequate', 'doubtful' or 'inadequate'. ${ }^{19}$ Second, the result of each study will be rated against the updated criteria for good measurement properties as either 'sufficient' (+), 'insufficient' (-) or 'indeterminate' (?). ${ }^{193}$ Third, the evidence for each measurement property will be summarised and the quality of the evidence for each measurement property will be graded as 'high', 'moderate', 'low' or 'very low', using the Grading of Recommendations Assessment, Development and Evaluation approach. ${ }^{24}$ Two researchers will independently participate in the data extraction process, and any disagreements will be resolved by a third reviewer. The results from different studies on measurement properties will be statistically pooled 
in a meta-analysis. Mean values of statistical analysis on each measurement property (eg, Cronbach's alpha and intraclass correlation coefficient) will be calculated from pooled data from methodologically similar studies and the results statistically summarised via meta-analysis into forest plots with estimates of heterogeneity (option A). The results of studies will be qualitatively summarised to achieve a result (option B).

Correlation between the two researcher's assessments will be calculated using Cohen's kappa. Inter-rater agreement for screening, study selection and data extraction will be calculated with the statistical program SPSS (V.24.0), using Cohen's kappa coefficient.

\section{Patient and public involvement}

Patients and the public were not involved in the design of this protocol.

\section{ETHICS AND DISSEMINATION}

No individual data are involved in this study. The results will be disseminated by publication in a peer-reviewed journal and presented at a relevant conference.

\section{DISCUSSION}

Although health professionals are often involved in the process of BBN, it remains a significant challenge.$^{56}$ The assessment of health professionals' BBN is increasingly used in research, teaching and clinical settings. ${ }^{12-16}$

$\mathrm{BBN}$ is an important clinical skill, and its evaluation with validated instruments is crucial. ${ }^{13}{ }^{14}$ In this regard, the assessment of BBN will allow us to facilitate and improve the process in clinical, teaching and research settings. ${ }^{16}$

Several validated questionnaires are now available for assessing the BBN process, and choosing the most appropriate instrument for assessing BBN is essential. ${ }^{13} 1417$ In this context, the main aim of this systematic review is to identify and appraise all the instruments assessing BBN and their measurement properties.

This systematic review aims to provide a comprehensive assessment of current evidence of instruments assessing the BBN process. In addition, assessing health professionals' skills in BBN may result in the improvement of the $\mathrm{BBN}$ process, increased patient satisfaction and in health professionals feeling more comfortable with treatment decisions.

\section{Strengths and limitations}

To the best of our knowledge, this will be the most up-todate systematic review of instruments assessing BBN in the healthcare setting. The results of this study will inform clinicians and researchers on the quality and the appropriateness for different populations of the instruments assessing BBN.

This review will be carried out following the COSMIN statement. In this context, COSMIN is one of the most widespread and comprehensive tools to critically appraise the measurement properties of existing outcome measures' instruments, and for selecting the most suitable instrument. ${ }^{1922}$

Although a meticulous search was conducted in several databases, it is possible that not all the instruments available for the measurement of BBN will be identified. However, the sensitive search strategy that was specifically designed to identify instruments for assessing BBN, and the double independent review process (ie, study screening and selection of eligible studies) will minimise this problem.

Contributors All authors made substantial contributions to the conception and design, piloted the inclusion criteria and provided direction on the data extraction and analysis. DG-S formulated the idea for the study. IL-L and MGG drafted the article. JPL-S critically revised the draft for important intellectual content. All authors agreed on the final version.

Funding The authors have not declared a specific grant for this research from any funding agency in the public, commercial or not-for-profit sectors.

Competing interests None declared.

Patient and public involvement Patients and/or the public were not involved in the design, or conduct, or reporting, or dissemination plans of this research.

Patient consent for publication Not required.

Provenance and peer review Not commissioned; externally peer reviewed.

Open access This is an open access article distributed in accordance with the Creative Commons Attribution Non Commercial (CC BY-NC 4.0) license, which permits others to distribute, remix, adapt, build upon this work non-commercially, and license their derivative works on different terms, provided the original work is properly cited, appropriate credit is given, any changes made indicated, and the use is non-commercial. See: http://creativecommons.org/licenses/by-nc/4.0/.

ORCID iD

Marina García-Gámez http://orcid.org/0000-0001-9953-9812

\section{REFERENCES}

1 Paul M. Breaking bad news. Acad Med 2018;93:1390.

2 Dean A, Willis S. The use of protocol in breaking bad news: evidence and ethos. Int J Palliat Nurs 2016;22:265-71.

3 Corey VR, Gwyn PG. Experiences of nurse practitioners in communicating bad news to cancer patients. J Adv Pract Oncol 2016;7:485-94.

4 Luz R, George A, Spitz E, et al. Breaking bad news in prenatal medicine: a literature review. J Reprod Infant Psychol 2017;35:14-31.

5 Fontes CMB, Menezes DVde, Borgato MH, et al. Communicating bad news: an integrative review of the nursing literature. Rev Bras Enferm 2017:70:1089-95.

6 Collini A, Parker H, Oliver A. Training for difficult conversations and breaking bad news over the phone in the emergency department. Emerg Med J 2020. doi:10.1136/emermed-2020-210141. [Epub ahead of print: 03 Dec 2020].

7 Camargo NC, Lima MGde, Brietzke E, et al. Teaching how to deliver bad news: a systematic review. Revista Bioética 2019;27:326-40.

8 Baran CN, Sanders JJ. Communication skills: delivering bad news, conducting a goals of care family meeting, and advance care planning. Prim Care 2019;46:353-72.

9 Matthews T, Baken D, Ross K. Single cases from multiple perspectives: a qualitative study comparing the experiences of patients, patients' caregivers, surgeons, and nurses when bad news is delivered about cancer. J Psychosoc Oncol Res Pract 2020;2:e32.

10 Banerjee SC, Manna R, Coyle N, et al. The implementation and evaluation of a communication skills training program for oncology nurses. Trans/ Behav Med 2017;7:615-23.

11 Boissy A, Windover AK, Bokar D, et al. Communication skills training for physicians improves patient satisfaction. J Gen Intern Med 2016;31:755-61.

12 Martis L, Westhues A. A synthesis of the literature on breaking bad news or truth telling: potential for research in India. Indian J Palliat Care 2013;19:2-11. 
13 Trickey AW, Newcomb AB, Porrey M, et al. Assessment of surgery residents' interpersonal communication skills: validation evidence for the communication assessment tool in a simulation environment. $J$ Surg Educ 2016;73:e19-27.

14 González-Cabrera M, Ortega-Martínez AR, Martínez-Galiano JM, et al. Design and validation of a questionnaire on communicating bad news in nursing: a pilot study. Int J Environ Res Public Health 2020;17. doi:10.3390/ijerph17020457. [Epub ahead of print: 10 Jan 2020].

15 Bumb M, Keefe J, Miller L, et al. Breaking bad news: an evidencebased review of communication models for oncology nurses. Clin J Oncol Nurs 2017;21:573-80.

16 Johnson J, Panagioti M. Interventions to improve the breaking of bad or difficult news by physicians, medical students, and interns/ residents: a systematic review and meta-analysis. Acad Med 2018:93:1400-12.

17 Prinsen CAC, Vohra S, Rose MR, et al. How to select outcome measurement instruments for outcomes included in a "Core Outcome Set" - a practical guideline. Trials 2016;17:449.

18 Singh MM, Agarwal RK. Breaking bad news in clinical setting: a systematic review. IJAR 2017;7:29-32.
19 Prinsen CAC, Mokkink LB, Bouter LM, et al. COSMIN guideline for systematic reviews of patient-reported outcome measures. Qual Life Res 2018;27:1147-57.

20 Moher D, Shamseer L, Clarke M, et al. Preferred reporting items for systematic review and meta-analysis protocols (PRISMA-P) 2015 statement. Syst Rev 2015;4:1.

21 McGowan J, Sampson M, Salzwedel DM, et al. PRESS Peer Review of Electronic Search Strategies: 2015 Guideline Statement. J Clin Epidemiol 2016;75:40-6.

22 Mokkink LB, de Vet HCW, Prinsen CAC, et al. COSMIN risk of bias checklist for systematic reviews of patient-reported outcome measures. Qual Life Res 2018;27:1171-9.

23 Terwee CB, Bot SDM, de Boer MR, et al. Quality criteria were proposed for measurement properties of health status questionnaires. J Clin Epidemiol 2007;60:34-42.

24 Guyatt GH, Oxman AD, Vist GE, et al. Grade: an emerging consensus on rating quality of evidence and strength of recommendations. $B M J$ 2008;336:924-6. 